Research Article

\title{
Solving Famous Nonlinear Coupled Equations with Parameters Derivative by Homotopy Analysis Method
}

\author{
Sohrab Effati, Hassan Saberi Nik, and Reza Buzhabadi \\ Department of Applied Mathematics, Ferdowsi University of Mashhad, P.O. Box 1159, \\ Mashhad 91779-48974, Iran \\ Correspondence should be addressed to Hassan Saberi Nik, saberi_hssn@yahoo.com and \\ Reza Buzhabadi, reza_bozhabadi@yahoo.com
}

Received 15 May 2011; Accepted 4 July 2011

Academic Editor: Shaher M. Momani

Copyright (C) 2011 Sohrab Effati et al. This is an open access article distributed under the Creative Commons Attribution License, which permits unrestricted use, distribution, and reproduction in any medium, provided the original work is properly cited.

\begin{abstract}
The homotopy analysis method (HAM) is employed to obtain symbolic approximate solutions for nonlinear coupled equations with parameters derivative. These nonlinear coupled equations with parameters derivative contain many important mathematical physics equations and reaction diffusion equations. By choosing different values of the parameters in general formal numerical solutions, as a result, a very rapidly convergent series solution is obtained. The efficiency and accuracy of the method are verified by using two famous examples: coupled Burgers and mKdV equations. The obtained results show that the homotopy perturbation method is a special case of homotopy analysis method.
\end{abstract}

\section{Introduction}

Fractional differential equations have gained importance and popularity during the past three decades or so, mainly due to its demonstrated applications in numerous seemingly diverse fields of science and engineering. For example, the nonlinear oscillation of earthquake can be modeled with fractional derivatives, and the fluid-dynamic traffic model with fractional derivatives can eliminate the deficiency arising from the assumption of continuum traffic flow. The differential equations with fractional order have recently proved to be valuable tools to the modeling of many physical phenomena [1,2]. This is because of the fact that the realistic modeling of a physical phenomenon does not depend only on the instant time, but also on the history of the previous time which can also be successfully achieved by using fractional calculus. Most nonlinear fractional equations do not have exact analytic solutions, so approximation and numerical techniques must be used. The Adomain decomposition method [3], the homotopy perturbation method [4], the variational iteration 
method [5], and other methods have been used to provide analytical approximation to linear and nonlinear problems. However, the convergence region of the corresponding results is rather small. In 1992, Liao employed the basic ideas of the homotopy in topology to propose a general analytic method for nonlinear problems, namely, homotopy analysis method [610]. This method has been successfully applied to solve many types of nonlinear problems in science and engineering, such as the viscous flows of non-Newtonian fluids [11], the KdV-type equations [12], finance problems [13], fractional Lorenz system [14], and delay differential equation [15]. The HAM contains a certain auxiliary parameter $h$ which provides us with a simple way to adjust and control the convergence region and rate of convergence of the series solution.

The HAM offers certain advantages over routine numerical methods. Numerical methods use discretization which gives rise to rounding off errors causing loss of accuracy and requires large computer memory and time. This computational method yields analytical solutions and has certain advantages over standard numerical methods. The HAM method is better since it does not involve discretization of the variables and hence is free from rounding off errors and does not require large computer memory or time.

In this paper, we extend the application of HAM to discuss the explicit numerical solutions of a type of nonlinear-coupled equations with parameters derivative in this form:

$$
\begin{array}{ll}
\frac{\partial^{\alpha} u}{\partial t^{\alpha}}=L_{1}(u, v)+N_{1}(u, v), & t>0, \\
\frac{\partial^{\beta} v}{\partial t^{\beta}}=L_{2}(u, v)+N_{2}(u, v), & t>0,
\end{array}
$$

where $L_{i}$ and $N_{i}(i=1,2)$ are the linear and nonlinear functions of $u$ and $v$, respectively, $\alpha$ and $\beta$ are the parameters that describe the order of the derivative. Different nonlinear coupled systems can be obtained when one of the parameters $\alpha$ or $\beta$ varies. The study of (1.1) is very necessary and significant because when $\alpha$ and $\beta$ are integers, it contains many important mathematical physics equations.

The paper has been organized as follows. Notations and basic definitions are given in Section 2. In Section 3 the homotopy analysis method is described. In Section 4 applying HAM for two famous coupled examples: Burgers and $\mathrm{mKdV}$ equations. Discussion and conclusions are presented in Section 5.

\section{Description on the Fractional Calculus}

Definition 2.1. A real function $f(t), t>0$ is said to be in the space $C_{\mu}, \mu \in R$ if there exists a real number $p>\mu$, such that $f(t)=t^{p} f_{1}(t)$ where $f_{1} \in(0, \infty)$, and it is said to be in the space $C_{n}^{\mu} 1$ if and only if $h(n) \in C_{\mu}, n \in N$. Clearly $C_{\mu} \subset C_{\nu}$ if $v \leq \mu$.

Definition 2.2. The Riemann-Liouville fractional integral operator $\left(J^{\alpha}\right)$ of order $\alpha \geq 0$, of a function $f \in C_{\mu}, \mu \geq-1$, is defined as

$$
\begin{gathered}
J^{\alpha} f(x)=\frac{1}{\Gamma(\alpha)} \int_{0}^{x}(x-t)^{\alpha-1} f(t) d t, \quad x>0 . \\
J^{0} f(x)=f(x) .
\end{gathered}
$$


$\Gamma(\alpha)$ is the well-known Gamma function. Some of the properties of the operator $J^{\alpha}$, which we will need here, are as follows.

$$
\begin{aligned}
& \text { For } f \in C_{\mu}, \mu \geq-1, \alpha, \beta \geq 0 \text { and } \gamma \geq-1 \\
& \qquad \begin{array}{r}
J^{\alpha} J^{\beta} f(x)=J^{\alpha+\beta} f(x), \\
J^{\alpha} J^{\beta} f(x)=J^{\beta} J^{\alpha} f(x), \\
J^{\alpha} t^{\gamma}=\frac{\Gamma(\gamma+1)}{\Gamma(\alpha+\gamma+1)} t^{\alpha+\gamma} .
\end{array}
\end{aligned}
$$

Definition 2.3. For the concept of fractional derivative, there exist many mathematical definitions [1, 16-19]. In this paper, the two most commonly used definitions: the Caputo derivative and its reverse operator Riemann-Liouville integral are adopted. That is because Caputo fractional derivative [1] allows the traditional assumption of initial and boundary conditions. The Caputo fractional derivative is defined as

$$
\begin{aligned}
D_{t}^{\alpha} u(x, t) & =\frac{\partial^{\alpha} u(x, t)}{\partial t^{\alpha}} \\
& = \begin{cases}\frac{1}{\Gamma(n-\alpha)} \int_{0}^{t}(t-\tau)^{n-\alpha-1} \frac{\partial^{n} u(x, t)}{\partial t^{n}} d \tau, & n-1<\alpha<n, \\
\frac{\partial^{n} u(x, t)}{\partial t^{n}}, & \alpha=n \in N .\end{cases}
\end{aligned}
$$

Here, we also need two basic properties about them:

$$
\begin{gathered}
D^{\alpha} J^{\alpha} f(x)=f(x), \\
J^{\alpha} D^{\alpha} f(x)=f(x)-\sum_{k=0}^{\infty} f^{(k)}\left(0^{+}\right) \frac{x^{k}}{k !}, \quad x>0 .
\end{gathered}
$$

Definition 2.4. The Mittag-Leffler function $E_{\alpha}(z)$ with $a>0$ is defined by the following series representation, valid in the whole complex plane:

$$
E_{\alpha}(z)=\sum_{n=0}^{\infty} \frac{z^{n}}{\Gamma(\alpha n+1)}, \quad \alpha>0, z \in \mathrm{C}
$$

\section{Basic Idea of HAM}

To describe the basic ideas of the HAM, we consider the operator form of (1.1):

$$
\begin{aligned}
& N\left[D_{t}^{\alpha} u(x, t)\right]=0, \quad t>0, \\
& N\left[D_{t}^{\beta} v(x, t)\right]=0, \quad t>0,
\end{aligned}
$$

where $N$ is nonlinear operator, $D_{t}^{\alpha}$ and $D_{t}^{\beta}$ stand for the fractional derivative and are defined as in (2.3), $t$ denotes an independent operator, and $u(x, t), v(x, t)$ are unknown functions. 
By means of generalizing the traditional homotopy method, Liao [6] constructs the so-called zero-order deformation equations:

$$
\begin{aligned}
& (1-q) L\left[\phi_{1}(x, t, q)-u_{0}(x, t)\right]=q h H(t) N\left[D_{t}^{\alpha} \phi_{1}(x, t, q)\right], \\
& (1-q) L\left[\phi_{2}(x, t, q)-v_{0}(x, t)\right]=q h H(t) N\left[D_{t}^{\beta} \phi_{2}(x, t, q)\right],
\end{aligned}
$$

where $q \in[0,1]$ is the embedding parameter, $h \neq 0$ is a non-zero auxiliary parameter, $H(t) \neq 0$ is an auxiliary function, $L$ is an auxiliary linear operator, $u_{0}(x, t), v_{0}(x, t)$ are initial guesses of $u(x, t), v(x, t)$ and $\phi_{1}(x, t, q), \phi_{2}(x, t, q)$ are two unknown functions, respectively. It is important that one has great freedom to choose auxiliary things in HAM. Obviously, when $q=0$ and $q=1$, the following holds:

$$
\begin{array}{ll}
\phi_{1}(x, t, 0)=u_{0}(x, t), & \phi_{1}(x, t, 1)=u(x, t), \\
\phi_{2}(x, t, 0)=v_{0}(x, t), & \phi_{2}(x, t, 1)=v(x, t),
\end{array}
$$

respectively. Thus, as $q$ increases from 0 to 1 , the solution $\phi_{1}(x, t, q), \phi_{2}(x, t, q)$ varies from the initial guess $u_{0}(x, t), v_{0}(x, t)$ to the solution $u(x, t), v(x, t)$. Expanding $\phi_{1}(x, t, q), \phi_{2}(x, t, q)$ in Taylor series with respect to $q$, we have

$$
\begin{aligned}
& \phi_{1}(x, t, q)=u_{0}(x, t)+\sum_{m=1}^{+\infty} u_{m}(x, t) q^{m} \\
& \phi_{2}(x, t, q)=v_{0}(x, t)+\sum_{m=1}^{+\infty} v_{m}(x, t) q^{m}
\end{aligned}
$$

where

$$
\begin{aligned}
& u_{m}(x, t)=\left.\frac{1}{m !} \frac{\partial^{m} \phi_{1}(x, t, q)}{\partial q^{m}}\right|_{q=0}, \\
& v_{m}(x, t)=\left.\frac{1}{m !} \frac{\partial^{m} \phi_{2}(x, t, q)}{\partial q^{m}}\right|_{q=0} .
\end{aligned}
$$

If the auxiliary linear operator, the initial guess, the auxiliary parameter $h$, and the auxiliary function are so properly chosen, the series (3.5) converges at $q=1$, then we have

$$
\begin{aligned}
& u(x, t)=u_{0}(x, t)+\sum_{m=1}^{+\infty} u_{m}(x, t), \\
& v(x, t)=v_{0}(x, t)+\sum_{m=1}^{+\infty} v_{m}(x, t),
\end{aligned}
$$


which must be one of solutions of original nonlinear equation, as proved by Liao [8]. As $h=-1$ and $H(\mathrm{t})=1,(3.2)$ and (3.3) become

$$
\begin{aligned}
& (1-q) L\left[\phi_{1}(x, t, q)-u_{0}(x, t)\right]+q N\left[\phi_{1}(x, t, q)\right]=0, \\
& (1-q) L\left[\phi_{2}(x, t, q)-u_{0}(x, t)\right]+q N\left[\phi_{2}(x, t, ; q)\right]=0,
\end{aligned}
$$

which is used mostly in the homotopy perturbation method [20], where as the solution obtained directly, without using Taylor series. According to the definition (3.6), the governing equation can be deduced from the zero-order deformation equation (3.2). Define the vector

$$
\vec{u}_{n}=\left\{u_{0}(x, t), u_{1}(x, t), \ldots, u_{n}(x, t)\right\}, \quad \vec{v}_{n}=\left\{v_{0}(x, t), v_{1}(x, t), \ldots, v_{n}(x, t)\right\}
$$

Differentiating equations (3.2) and (3.3) $m$ times with respect to the embedding parameter $q$ and then setting $q=0$ and finally dividing them by $m !$, we have the so-called $m$ th-order deformation equation:

$$
\begin{aligned}
& L\left[u_{m}(x, t)-x_{m} u_{m-1}(x, t)\right]=h H(t) R_{1, m}\left(\vec{u}_{m-1}\right), \\
& L\left[v_{m}(x, t)-x_{m} v_{m-1}(x, t)\right]=h H(t) R_{2, m}\left(\vec{v}_{m-1}\right),
\end{aligned}
$$

where

$$
\begin{gathered}
R_{1, m}\left(\vec{u}_{m-1}\right)=\left.\frac{1}{(m-1) !} \frac{\partial^{m-1} D_{t}^{\alpha}\left[\phi_{1}(x, t, q)\right]}{\partial q^{m-1}}\right|_{q=0}, \\
R_{2, m}\left(\vec{v}_{m-1}\right)=\left.\frac{1}{(m-1) !} \frac{\partial^{m-1} D_{t}^{\beta}\left[\phi_{2}(x, t, q)\right]}{\partial q^{m-1}}\right|_{q=0}, \\
x_{m}= \begin{cases}0, & m \leqslant 1, \\
1, & m>1 .\end{cases}
\end{gathered}
$$

Applying the Riemann-Liouville integral operator $J^{\alpha}, J^{\beta}$ on both side of (3.10), we have

$$
\begin{aligned}
& u_{m}(x, t)=\chi_{m} u_{m-1}(x, t)-\chi_{m} \sum_{i=0}^{n-1} u_{m-1}^{i}\left(0^{+}\right) \frac{t^{i}}{i !}+h H(t) J^{\alpha} R_{1, m}\left(\vec{u}_{m-1}\right), \\
& v_{m}(x, t)=\chi_{m} v_{m-1}(x, t)-\chi_{m} \sum_{i=0}^{n-1} v_{m-1}^{i}\left(0^{+}\right) \frac{t^{i}}{i !}+h H(t) J^{\beta} R_{2, m}\left(\vec{v}_{m-1}\right) .
\end{aligned}
$$

It should be emphasized that $u_{m}(x, t), v_{m}(x, t)$ for $m \geqslant 1$ is governed by the linear equation (3.10), under the linear boundary conditions that come from original problem, which can be easily solved by symbolic computation software such as MATLAB. For the convergence 
of the above method we refer the reader to Liao's work. Liao [7] proved that, as long as a series solution given by the homotopy analysis method converges, it must be one of exact solutions. So, it is important to ensure that the solution series is convergent. Note that the solution series contain the auxiliary parameter $h$, which we can choose properly by plotting the so-called $h$-curves to ensure solution series converge.

Remark 3.1. The parameters $\alpha$ and $\beta$ can be arbitrarily chosen as, integer or fraction, bigger or smaller than 1 . When the parameters are bigger than 1 , we will need more initial and boundary conditions such as $u_{0}^{\prime}(x, 0), u_{0}^{\prime \prime}(x, 0), \ldots$ and the calculations will become more complicated correspondingly. In order to illustrate the problem and make it convenient for the readers, we only confine the parameters to $[0,1]$ to discuss.

\section{Application}

\subsection{The Nonlinear Coupled Burgers Equations with Parameters Derivative}

In order to illustrate the method discussed above, we consider the nonlinear coupled Burgers equations with parameters derivative in an operator form:

$$
\begin{array}{ll}
D_{t}^{\alpha} u-L_{x x} u-2 u L_{x} u+L_{x} u v=0, & (0<\alpha \leq 1), \\
D_{t}^{\beta} v-L_{x x} v-2 v L_{x} v+L_{x} u v=0, & (0<\beta \leq 1),
\end{array}
$$

where $t>0, L_{x}=\partial / \partial x$ and the fractional operators $D_{t}^{\alpha}$ and $D_{t}^{\beta}$ are defined as in (2.3). Assuming the initial value as

$$
u(x, 0)=\sin x, \quad v(x, 0)=\sin x .
$$

The exact solutions of (4.1) for the special case: $\alpha=\beta=1$ are

$$
u(x, t)=e^{-t} \sin x, \quad v(x, t)=e^{-t} \sin x .
$$

For application of homotopy analysis method, in view of (4.1) and the initial condition given in (4.2), it is convenient to choose

$$
u_{0}(x, t)=\sin x, \quad v_{0}(x, t)=\sin x,
$$

as the initial approximate of (4.1). We choose the linear operators

$$
\begin{aligned}
& L_{1}\left[\phi_{1}(x, t, q)\right]=D_{t}^{\alpha}\left[\phi_{1}(x, t, q)\right], \\
& L_{2}\left[\phi_{2}(x, t, q)\right]=D_{t}^{\beta}\left[\phi_{2}(x, t, q)\right],
\end{aligned}
$$


with the property $L(c)=0$ where $c$ is constant of integration. Furthermore, we define a system of nonlinear operators as

$$
\begin{aligned}
N_{1}\left[\phi_{i}(x, t, q)\right]= & D_{t}^{\alpha}\left[\phi_{1}(x, t, q)\right]-\frac{\partial^{2} \phi_{1}(x, t, q)}{\partial x^{2}}-2 \phi_{1}(x, t, q) \frac{\partial \phi_{1}(x, t, q)}{\partial x} \\
& +\frac{\partial\left[\phi_{1}(x, t, q) \phi_{2}(x, t, q)\right]}{\partial x} \\
N_{2}\left[\phi_{i}(x, t, q)\right]= & D_{t}^{\beta}\left[\phi_{2}(x, t, q)\right]-\frac{\partial^{2} \phi_{2}(x, t, q)}{\partial x^{2}}-2 \phi_{2}(x, t, q) \frac{\partial \phi_{2}(x, t, q)}{\partial x} \\
& +\frac{\partial\left[\phi_{1}(x, t, q) \phi_{2}(x, t, q)\right]}{\partial x} .
\end{aligned}
$$

We construct the zeroth-order and the $m$ th-order deformation equations where

$$
\begin{aligned}
& R_{1, m}\left(\vec{u}_{m-1}\right)=D_{t}^{\alpha}\left[u_{m-1}\right]-\left(u_{m-1}\right)_{x x}-2 \sum_{k=0}^{m-1} u_{k}\left(u_{m-1-k}\right)_{x}+\left(\sum_{k=0}^{m-1} u_{k} v_{m-k-1}\right)_{x} \\
& R_{2, m}\left(\vec{v}_{m-1}\right)=D_{t}^{\beta}\left[v_{m-1}\right]-\left(v_{m-1}\right)_{x x}-2 \sum_{k=0}^{m-1} v_{k}\left(v_{m-1-k}\right)_{x}+\left(\sum_{k=0}^{m-1} u_{k} v_{m-k-1}\right)_{x}
\end{aligned}
$$

We start with an initial approximation $u(x, 0)=\sin (x), v(x, 0)=\sin (x)$, thus we can obtain directly the other components as

$$
\begin{aligned}
u_{1}= & \frac{h t^{a} \sin (x)}{\Gamma(a+1)} \\
u_{2}= & \frac{-\sin x}{a^{2} \Gamma(b+1) \Gamma(a)^{2} \Gamma(a+(1 / 2))} \\
& \times\left[a^{2} \Gamma(b+1) \Gamma(a)^{2} \Gamma\left(a+\frac{1}{2}\right)-a^{2} \Gamma(b+1) \Gamma(a)^{2}\right. \\
& \Gamma\left(a+\frac{1}{2}\right) h+h a \Gamma(a) \Gamma(2 a+1) t^{a} \Gamma(b+1)+h^{2} a \Gamma(a) \Gamma\left(a+\frac{1}{2}\right) t^{a} \Gamma(b+1)+2 h^{2} a \Gamma(a) \\
& \Gamma\left(a+\frac{1}{2}\right) t^{(b+a)} \cos (x)-2 h^{2} t^{(2 a)} \cos (x) \Gamma(b+1) \Gamma\left(a+\frac{1}{2}\right) \\
& \left.+h^{2} t^{(2 a)} a^{2} \Gamma(b+1) \Gamma(a)^{2}\right]
\end{aligned}
$$




$$
\begin{aligned}
& v_{1}=\frac{h t^{b} \sin (x)}{\Gamma(b+1)}, \\
& v_{2}=\frac{-\sin x}{\left(b^{2} \Gamma(a+1) \Gamma(b)^{2} \Gamma(b+(1 / 2))\right)} \\
& \quad \times\left[b^{2} \Gamma(a+1) \Gamma(b)^{2} \Gamma(2 b+1) h+h b \Gamma(b),\right. \\
& \quad \Gamma(2 b+1) t^{b} \Gamma(a+1)+h^{2} b \Gamma(b) \Gamma(2 b+1) t^{b} \Gamma(b+1)+2 h^{2} b \Gamma(b) \Gamma(2 b+1), \\
& t^{(a+b)} \cos (x)-2 h^{2} t^{(2 b)} \cos (x) \Gamma(a+1) \Gamma(2 b+1)+h^{2}\left(\frac{1}{t}\right)^{(-b)} t^{b} b^{2} \Gamma(a+1), \\
& \left.\Gamma(b)^{2}\right]
\end{aligned}
$$

The absolute error of the 6th-order HAM and exact solution with $h=-1$ as shown in Figure 1 . Also the absolute errors $\left|u(t)-\phi_{6}(t)\right|$ have been calculated in Table 1. Figure 2 shows the numerical solutions of the nonlinear coupled Burgers equations with parameters derivative with $h=-1, \alpha=\beta=1$. Figure 3 shows the explicit numerical solutions with $h=-1, \alpha=1 / 4$, and $\beta=1 / 3$ at $t=0.02$.

As suggested by Liao [7], the appropriate region for $h$ is a horizontal line segment. We can investigate the influence of $h$ on the convergence of the solution series gevin by the HAM, by plotting its curve versus $h$, as shown in Figure 4 .

Remark 4.1. This example has been solved using homotopy perturbation method [21]. The graphs drawn and tables by $h=-1$ are in excellent agreement with that graphs drawn with HPM.

\subsection{The Nonlinear Coupled mKdV Equations with Parameters Derivative}

In order to illustrate the method discussed above, we consider the nonlinear coupled mKdV equations with parameters derivative in an operator form:

$$
\begin{gathered}
D_{t}^{\alpha} u-\frac{1}{2} u_{x x x}+3 u^{2} u_{x}-\frac{3}{2} v_{x x}-3(u v)_{x}+3 \lambda u_{x}=0, \\
D_{t}^{\beta} v+v_{x x x}+3 v v_{x}+3 u_{x} v_{x}-3 u^{2} v_{x}-3 \lambda v_{x}=0
\end{gathered}
$$

with the initial conditions,

$$
u(x, 0)=\frac{b}{2 k}+k \tanh (k x), \quad v(x, 0)=\frac{\lambda}{2}\left(1+\frac{k}{b}\right)+b \tanh (k x) .
$$




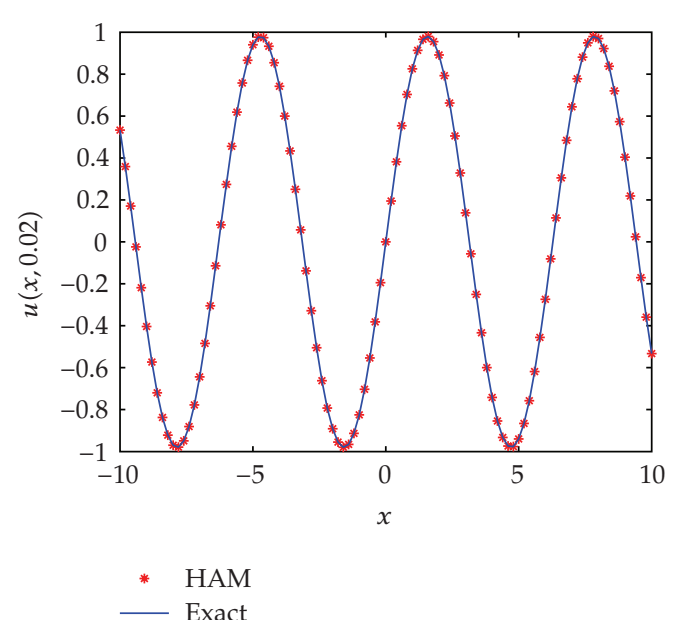

(a)

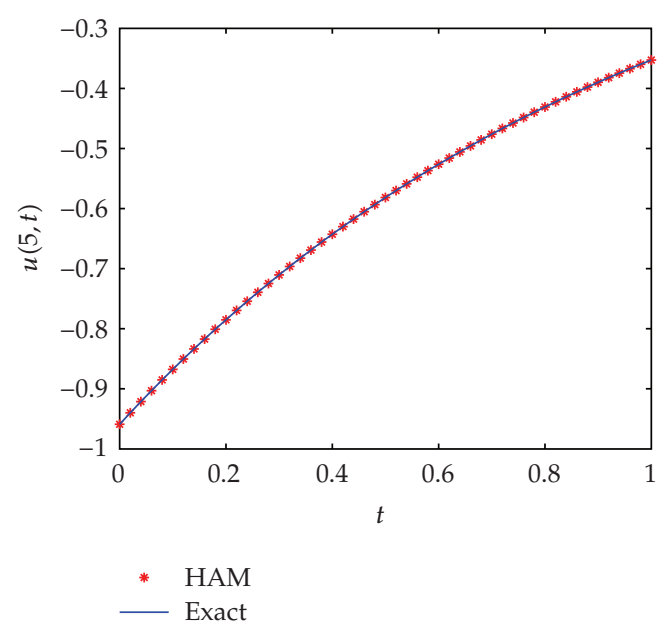

(b)

Figure 1: The comparison of the 6th-order HAM and exact solution with $h=-1, \alpha=\beta=1$.

Table 1: The comparison of the results of the HAM $(h=-1)$ and exact solution for the $u(x, t), \alpha=\beta=1$.

\begin{tabular}{lcccc}
\hline$x$ & $t$ & $\phi_{6}$ & $u(x, t)$ & Realtive error \\
\hline 10 & 0.01 & $-5.3861 \mathrm{e}-001$ & -0.9002497662 & $4.5253 e \times 10^{-13}$ \\
10 & 0.02 & $-5.3325 \mathrm{e}-001$ & -0.8912921314 & $1.4459 \times 10^{-11}$ \\
10 & 0.03 & $-5.2794 \mathrm{e}-001$ & -0.8824236265 & $1.0962 \times 10^{-10}$ \\
5 & 0.01 & -0.9493828183 & -0.9493828187 & $7.9781 \times 10^{-13}$ \\
5 & 0.02 & -0.9399363019 & -0.9399363019 & $2.5486 \times 10^{-11}$ \\
5 & 0.03 & -0.9305837792 & -0.9305837793 & $1.9322 \times 10^{-10}$ \\
-2 & 0.01 & -0.5386080102 & -0.5386080104 & $7.5651 \times 10^{-13}$ \\
-2 & 0.02 & -0.5332487712 & -0.5332487712 & $2.4167 \times 10^{-11}$ \\
-2 & 0.03 & -0.5279428571 & -0.5279428572 & $1.8322 \times 10^{-10}$ \\
\hline
\end{tabular}

As we know, when $\alpha=\beta=1$ (4.9) has the kink-type soliton solutions

$$
\begin{gathered}
u(x, t)=\frac{b}{2 k}+k \tanh (k \xi), \\
v(x, t)=\frac{\lambda}{2}\left(1+\frac{k}{b}\right)+b \tanh (k \xi),
\end{gathered}
$$

constructed by Fan [22], where $\xi=x+(1 / 4)\left(-4 k^{2}-6 \lambda+6 k \lambda / b+3 b^{2} / k^{2}\right) t, k \neq 0$, and $b \neq 0$. For application of homotopy analysis method, in view of (4.9) and the initial condition given in (4.10), it in convenient to choose

$$
u(x, 0)=\frac{b}{2 k}+k \tanh (k x), \quad v(x, 0)=\frac{l}{2}\left(1+\frac{k}{b}\right)+b \tanh (k x),
$$


Table 2: The comparison of the results of the HAM $(h=-1)$ and exact solution for the $u(x, t), \alpha=\beta=1$.

\begin{tabular}{lccc}
\hline$x$ & $t$ & $\mid \sum_{i=0}^{5} u(i)-$ Exact $\mid$ & $\mid \sum_{i=0}^{5} v(i)-$ Exact $\mid$ \\
\hline-15 & 0.002 & $8.0672 \times 10^{-8}$ & $2.4426 \times 10^{-8}$ \\
-12 & 0.002 & $5.9539 \times 10^{-7}$ & $1.8038 \times 10^{-7}$ \\
-6 & 0.002 & $3.0257 \times 10^{-6}$ & $1.3270 \times 10^{-6}$ \\
6 & 0.002 & $3.0263 \times 10^{-6}$ & $1.3481 \times 10^{-6}$ \\
12 & 0.002 & $5.9550 \times 10^{-7}$ & $1.7722 \times 10^{-7}$ \\
15 & 0.002 & $8.0686 \times 10^{-8}$ & $2.3998 \times 10^{-8}$ \\
\hline
\end{tabular}

as the initial approximate of (4.10). We choose the linear operators

$$
\begin{aligned}
& L_{1}\left[\phi_{1}(x, t, q)\right]=D_{t}^{\alpha}\left[\phi_{1}(x, t, q)\right], \\
& L_{2}\left[\phi_{2}(x, t, q)\right]=D_{t}^{\beta}\left[\phi_{2}(x, t, q)\right],
\end{aligned}
$$

with the property $L(c)=0$ where $c$ is constant of integration. Furthermore, we define a system of nonlinear operators as

$$
\begin{aligned}
N_{1}\left[\phi_{i}(x, t, q)\right]= & D_{t}^{\alpha}\left[\phi_{1}(x, t, q)\right]-\frac{1}{2} \frac{\partial^{3} \phi_{1}(x, t, q)}{\partial x^{3}}+3 \phi_{1}(x, t, q)^{2} \frac{\partial \phi_{1}(x, t, q)}{\partial x}, \\
& -\frac{3}{2} \frac{\partial^{2} \phi_{2}(x, t, q)}{\partial x^{2}}-3 \frac{\partial\left[\phi_{1}(x, t, q) \phi_{2}(x, t, q)\right]}{\partial x}+3 \lambda \frac{\partial \phi_{1}(x, t, q)}{\partial x}, \\
N_{2}\left[\phi_{i}(x, t, q)\right]= & D_{t}^{\beta}\left[\phi_{2}(x, t, q)\right]+\frac{\partial^{3} \phi_{2}(x, t, q)}{\partial x^{3}}+3 \phi_{2}(x, t, q) \frac{\partial \phi_{2}(x, t, q)}{\partial x}, \\
& +3 \frac{\partial \phi_{1}(x, t, q)}{\partial x} \frac{\partial \phi_{2}(x, t, q)}{\partial x}-3 \phi_{1}(x, t, q)^{2} \frac{\partial \phi_{2}(x, t, q)}{\partial x}-3 \curlywedge \frac{\partial \phi_{2}(x, t, q)}{\partial x} .
\end{aligned}
$$

We construct the zeroth-order and the $m$ th-order deformation equations where

$$
\begin{aligned}
R_{1, m}\left(\vec{u}_{m-1}\right)= & D_{t}^{\alpha}\left[u_{m-1}\right]-\frac{1}{2}\left(u_{m-1}\right)_{x x x}+3 \sum_{i=0}^{m-1} u_{i} \sum_{k=0}^{m-1-i} u_{k}\left(v_{m-1-i-k}\right)_{x}, \\
& -\frac{3}{2}\left(v_{m-1}\right)_{x x}-3\left(\sum_{k=0}^{m-1} u_{k} v_{m-k-1}\right)_{x}+3 \lambda\left(u_{m-1}\right)_{x} \\
R_{2, m}\left(\vec{v}_{m-1}\right)= & D_{t}^{\beta}\left[v_{m-1}\right]+\left(v_{m-1}\right)_{x x x}+3 \sum_{k=0}^{m-1} v_{k}\left(v_{m-1-k}\right)_{x}+3 \sum_{k=0}^{m-1}\left(u_{k}\right)_{x}\left(v_{m-k-1}\right)_{x}, \\
& -3 \sum_{i=0}^{m-1} u_{i} \sum_{k=0}^{m-1-i} u_{k}\left(v_{m-1-i-k}\right)_{x}-3 \lambda\left(v_{m-1}\right)_{x} .
\end{aligned}
$$


We start with an initial approximation $u(x, 0)=(b / 2 k)+k \tanh (k x), v(x, 0)=(\lambda / 2)(1+$ $(k / b))+b \tanh (k x)$, with $k=0.1, b=1, k=1 / 3$, thus we can obtain directly the other components as follows:

$$
\begin{aligned}
& u_{1}=\frac{1177}{1620} h t^{a} \frac{-1+\tanh ((1 / 3) x)^{2}}{\Gamma(a+1)} \\
& u_{2}=\frac{1}{437400}-656100 \Gamma(a+b+1) a^{2} \Gamma(b+1) \Gamma(a)^{2} \Gamma(2 a+1)-145800, \\
& \Gamma(a+b+1) a^{2} \Gamma(b+1) \Gamma(a)^{2} \Gamma(2 \mathrm{a}+1) \tanh \left(\frac{1}{3} x\right)-656100 \Gamma(a+b+1) a^{2}, \\
& \Gamma(b+1) \Gamma(a)^{2} \Gamma(2 a+1) h-145800 \Gamma(a+b+1) a^{2} \Gamma(b+1) \Gamma(a)^{2} \Gamma(2 a+1), \\
& h \tanh \left(\frac{1}{3} x\right)-327510 h^{2} t^{(b+a)} a^{2} \Gamma(b+1) \Gamma(a)^{2} \Gamma(2 a+1)+1310040 h^{2} t^{(b+a)}, \\
& a^{2} \Gamma(b+1) \Gamma(a)^{2} \Gamma(2 a+1) \tanh \left(\frac{1}{3} x\right)^{2}-982530 h^{2} t^{(b+a)} a^{2} \Gamma(b+1) \Gamma(a)^{2}, \\
& \Gamma(2 a+1) \tanh \left(\frac{1}{3} x\right)^{4}-317790 h \Gamma(a+b+1) a \Gamma(a) \Gamma(2 a+1) t^{a} \Gamma(b+1), \\
& +\cdots \\
& \vdots \\
& v_{1}=\frac{1213}{540} h t^{b} \frac{-1+\tanh ((1 / 3) x)^{2}}{\Gamma(b+1)} \\
& v_{2}=\frac{1}{145800}-145800 b^{2} \Gamma(a+1) \Gamma(b)^{2} \Gamma(2 b+1) h \tanh \left(\frac{1}{3} x\right)-145800 b^{2}, \\
& \Gamma(a+1) \Gamma(b)^{2} \Gamma(2 b+1) \tanh \left(\frac{1}{3} x\right)-9720 b^{2} \Gamma(a+1) \Gamma(b)^{2} \Gamma(2 b+1) h, \\
& -327510 h b \Gamma(b) \Gamma(2 b+1) t^{b} \Gamma(a+1)-327510 h^{2} b \Gamma(b) \Gamma(2 b+1) t^{b} \Gamma(a+1), \\
& +317790 h^{2} b \Gamma(b) \Gamma(2 b+1) t^{(b+a)}-635580 h^{2} b \Gamma(b) \Gamma(2 b+1) t^{(b+a)} ， \\
& \tanh \left(\frac{1}{3} x\right)^{2}-282480 h^{2} b \Gamma(b) \Gamma(2 b+1) t^{(b+a)} \tanh \left(\frac{1}{3} x\right)^{3}+141240 h^{2} b, \\
& \tanh \left(\frac{1}{3} x\right)^{4}+\cdots
\end{aligned}
$$




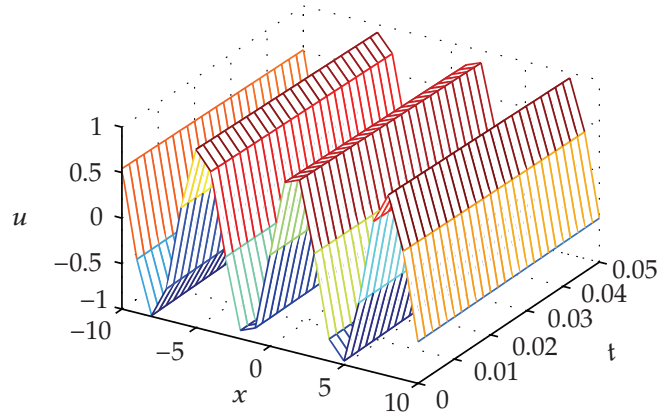

(a)

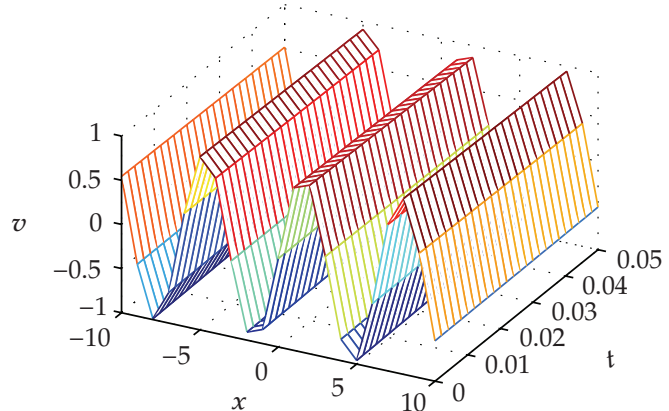

(b)

Figure 2: Explicit numerical solutions with $h=-1, \alpha=\beta=1$.

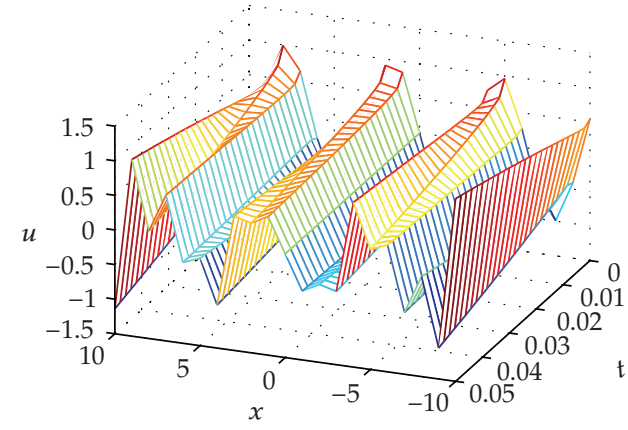

(a)

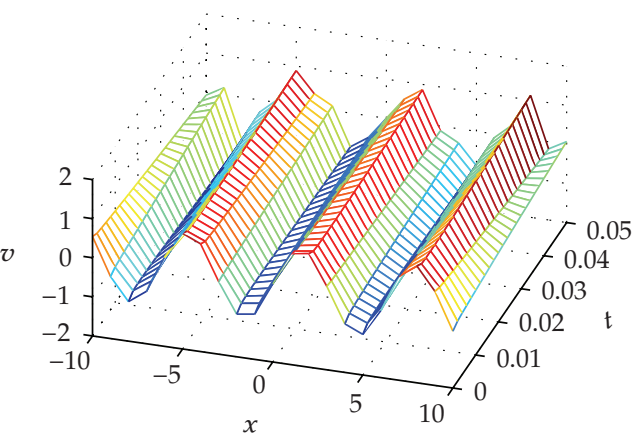

(b)

Figure 3: Explicit numerical solutions with $h=-1, \alpha=1 / 4$, and $\beta=1 / 3$.

The absolute error of the 6th-order HAM and exact solution with $h=-1$ as shown in Figure 5. Also the absolute errors $\left|u(t)-\phi_{6}(t)\right|$ have been calculated for in Table 2. Figure 6 shows the numerical solutions of the nonlinear coupled Burgers equations with parameters derivative with $h=-1, \alpha=\beta=1$. Figure 7 shows the explicit numerical solutions with $h=-1$, $\alpha=1 / 2$, and $\beta=2 / 3$ at $t=0.002$.

As suggested by Liao [7], the appropriate region for $h$ is a horizontal line segment. We can investigate the influence of $h$ on the convergence of the solution series gevin by the HAM, by plotting its curve versus $h$, as shown in Figure 8.

Remark 4.2. This example has been solved using homotopy perturbation method [21]. The graphs drawn and tables by $h=-1$ are in excellent agreement with those graphs drawn with HPM.

\section{Conclusion}

In this paper, based on the symbolic computation MATLAB, the HAM is directly extended to derive explicit and numerical solutions of the nonlinear coupled equations with parameters 


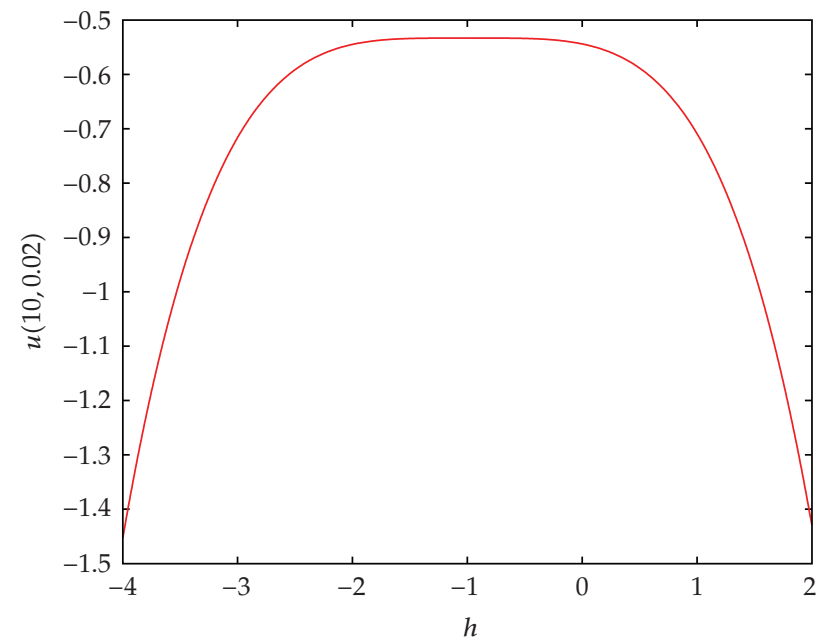

Figure 4: The $h$-curves obtained from the 5-order HAM approximate solution.

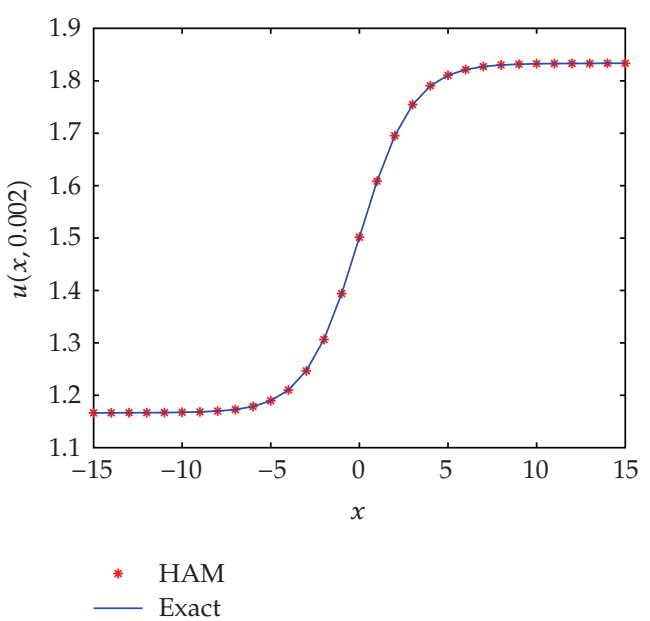

(a)

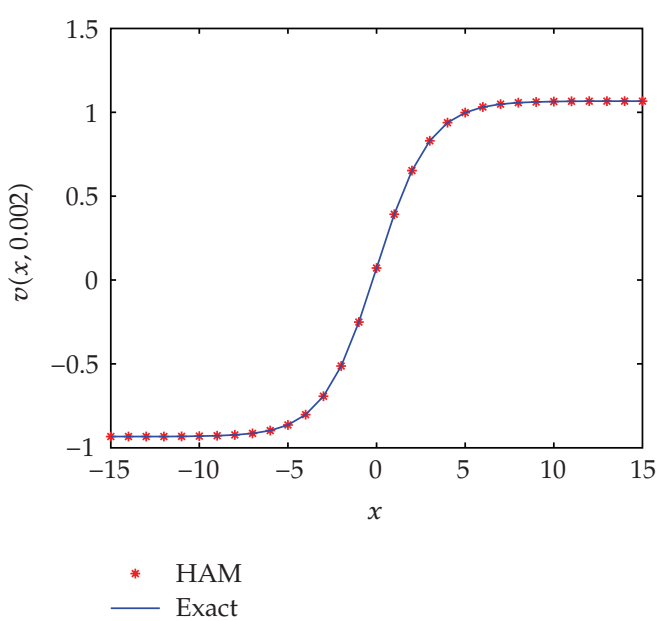

(b)

Figure 5: The comparison of the 6th-order HAM and exact solution with $=-1, \alpha=\beta=1, \lambda=0.1, b=1$, and $k=1 / 3$.

derivative. HAM provides us with a convenient way to control the convergence of approximation series by adapting $h$, which is a fundamental qualitative difference in analysis between HAM and other methods. The obtained results demonstrate the reliability of the HAM and its wider applicability to fractional differential equation. It, therefore, provides more realistic series solutions that generally converge very rapidly in real physical problems. MATLAB has been used for computations in this paper. 


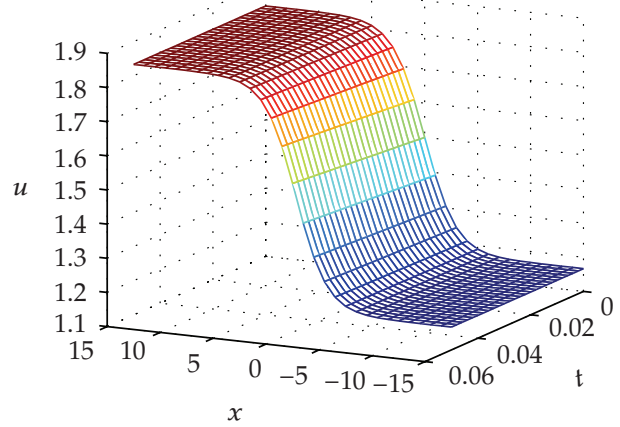

(a)

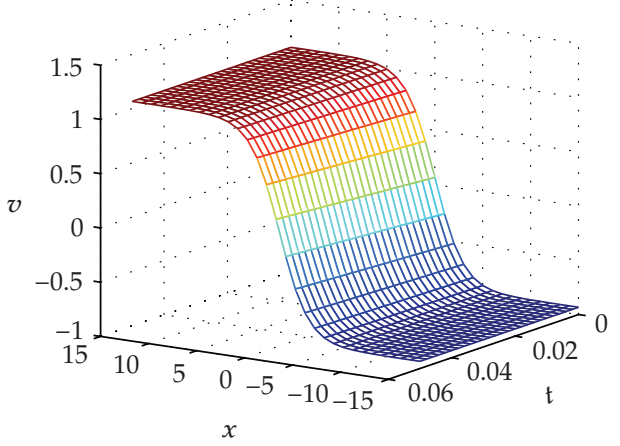

(b)

Figure 6: Explicit numerical solutions with $h=-1, \alpha=\beta=1, \lambda=0.1, b=1$, and $k=1 / 3$.

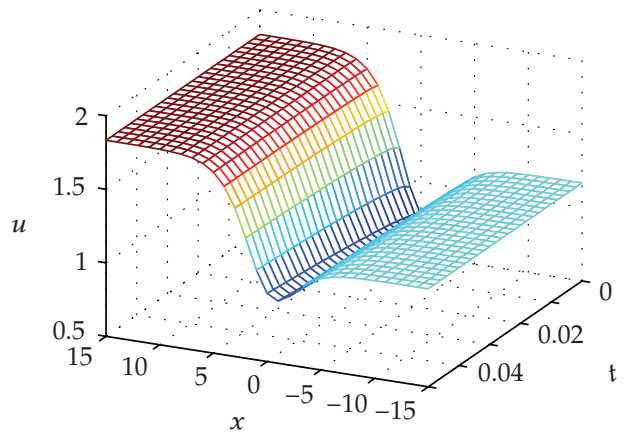

(a)

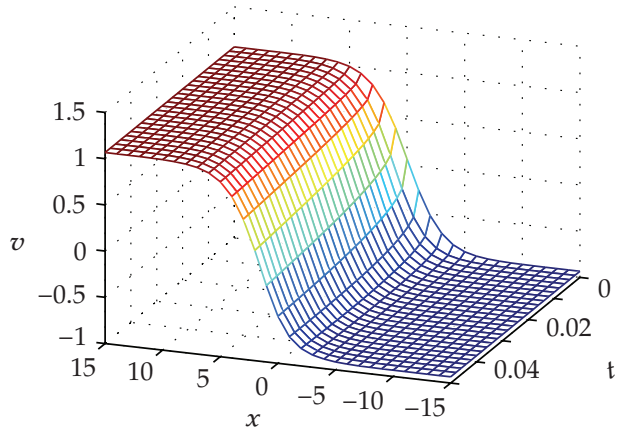

(b)

Figure 7: Explicit numerical solutions with $h=-1, \alpha=1 / 2, \beta=2 / 3, \lambda=0.1, b=1$, and $k=1 / 3$.

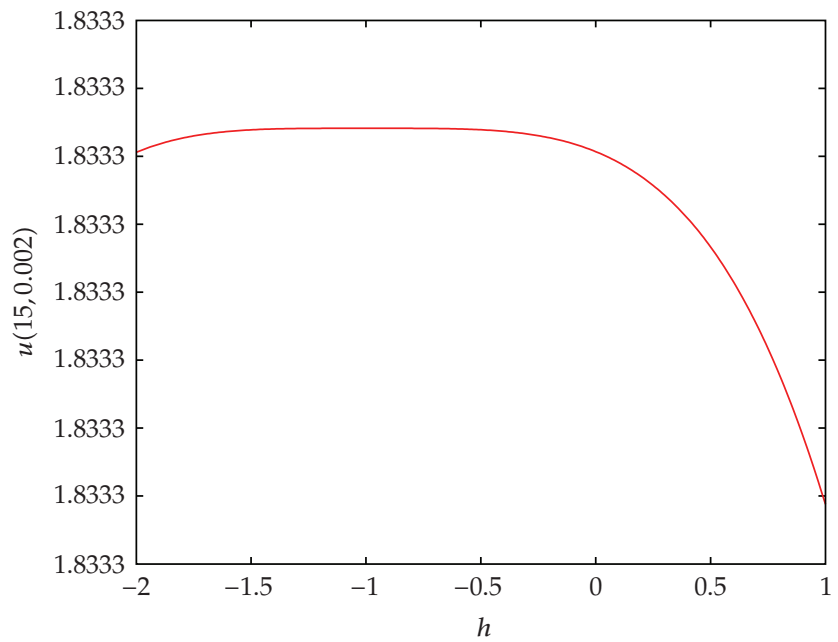

Figure 8: The $h$-curves obtained from the 5-order HAM approximate solution. 
International Journal of Differential Equations

\section{References}

[1] M. Caputo, "Linear models of dissipation whose Q is almost frequency independent, part II," Geophysical Journal of the Royal Astronomical Society, vol. 13, no. 5, pp. 529-539, 1967.

[2] K. B. Oldham and J. Spanier, The Fractional Calculus, Academic Press, New York, NY, USA, 1974.

[3] S. Momani and N. Shawagfeh, "Decomposition method for solving fractional Riccati differential equations," Applied Mathematics and Computation, vol. 182, no. 2, pp. 1083-1092, 2006.

[4] Z. Odibat and S. Momani, "Modified homotopy perturbation method: application to quadratic Riccati differential equation of fractional order," Chaos, Solitons and Fractals, vol. 36, no. 1, pp. 167-174, 2008.

[5] Z. Odibat and S. Momani, "Application of variation iteration method to nonlinear differential equations of fractional order," International Journal of Nonlinear Sciences and Numerical Simulation, vol. 1, no. 7, pp. 15-27, 2006.

[6] S. J. Liao, The proposed homotopy analysis technique for the solution of nonlinear problems, Ph.D. thesis, Shanghai Jiao Tong University, 1992.

[7] S. Liao, Beyond Perturbation: Introduction to the Homotopy Analysis Method, CRC Series: Modern Mechanics and Mathematics, Chapman \& Hall/CRC, Boca Raton, Fla, USA, 2003.

[8] S. Liao, "On the homotopy anaylsis method for nonlinear problems," Applied Mathematics and Computation, vol. 147, pp. 499-513, 2004.

[9] S. Liao, "Comparison between the homotopy analysis method and homotopy perturbation method," Applied Mathematics and Computation, vol. 169, no. 2, pp. 1186-1194, 2005.

[10] S. Liao, "Homotopy analysis method: a new analytical technique for nonlinear problems," Journal of Communications in Nonlinear Science and Numerical Simulation, vol. 2, no. 2, pp. 95-100, 1997.

[11] T. Hayat, M. Khan, and M. Ayub, “On non-linear flows with slip boundary condition," Zeitschrift für Angewandte Mathematik und Physik, vol. 56, no. 6, pp. 1012-1029, 2005.

[12] S. Abbasbandy and F. S. Zakaria, "Soliton solutions for the 5th-order KdV equation with the homotopy analysis method," Nonlinear Dynamics, vol. 51, no. 1-2, pp. 83-87, 2008.

[13] S. P. Zhu, "An exact and explicit solution for the valuation of American put options," Quantitative Finance, vol. 6, no. 3, pp. 229-242, 2006.

[14] A. K. Alomari, M. S. M. Noorani, R. Nazar, and C. P. Li, “Homotopy analysis method for solving fractional Lorenz system," Communications in Nonlinear Science and Numerical Simulation, vol. 15, no. 7, pp. 1864-1872, 2010.

[15] A. K. Alomari, M. S. M. Noorani, and R. Nazar, "Solution of delay differential equation by means of homotopy analysis method," Acta Applicandae Mathematicae, vol. 108, no. 2, pp. 395-412, 2009.

[16] B. J. West, M. Bologna, and P. Grigolini, Physics of Fractal Operators, Institute for Nonlinear Science, Springer, New York, NY, USA, 2003.

[17] K. S. Miller and B. Ross, An Introduction to the Fractional Calculus and Fractional Differential Equations, A Wiley-Interscience Publication, John Wiley \& Sons, New York, NY, USA, 1993.

[18] S. G. Samko, A. A. Kilbas, and O. I. Marichev, Fractional Integrals and Derivatives, Gordon and Breach Science Publishers, Yverdon, Switzerland, 1993.

[19] I. Podlubny, Fractional Differential Equations, vol. 198 of Mathematics in Science and Engineering, Academic Press, San Diego, Calif, USA, 1999.

[20] J. H. He, "Homotopy perturbation technique," Computer Methods in Applied Mechanics and Engineering, vol. 178, no. 3-4, pp. 257-262, 1999.

[21] Y. Chen and H. An, "Homotopy perturbation method for a type of nonlinear coupled equations with parameters derivative," Applied Mathematics and Computation, vol. 204, no. 2, pp. 764-772, 2008.

[22] E. G. Fan, "Soliton solutions for a generalized Hirota-Satsuma coupled KdV equation and a coupled MKdV equation," Physics Letters. A, vol. 282, no. 1-2, pp. 18-22, 2001. 


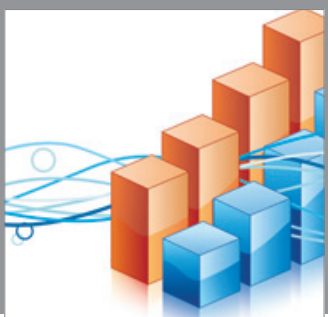

Advances in

Operations Research

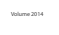

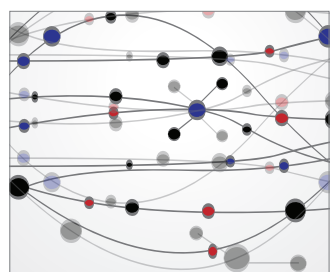

\section{The Scientific} World Journal
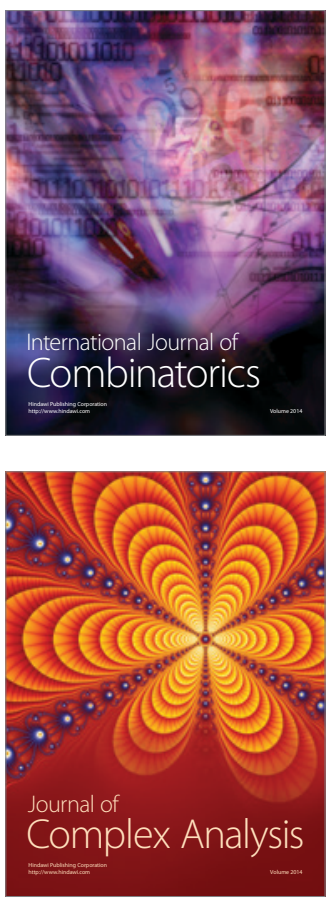

International Journal of

Mathematics and

Mathematical

Sciences
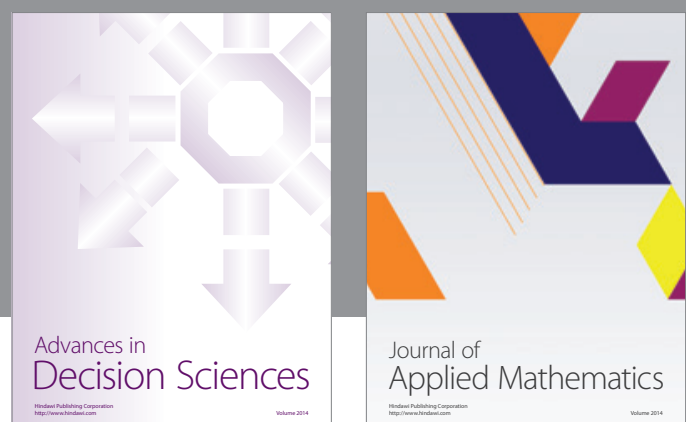

Journal of

Applied Mathematics
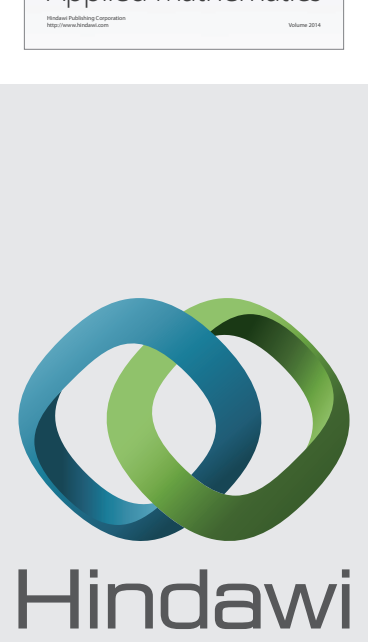

Submit your manuscripts at http://www.hindawi.com
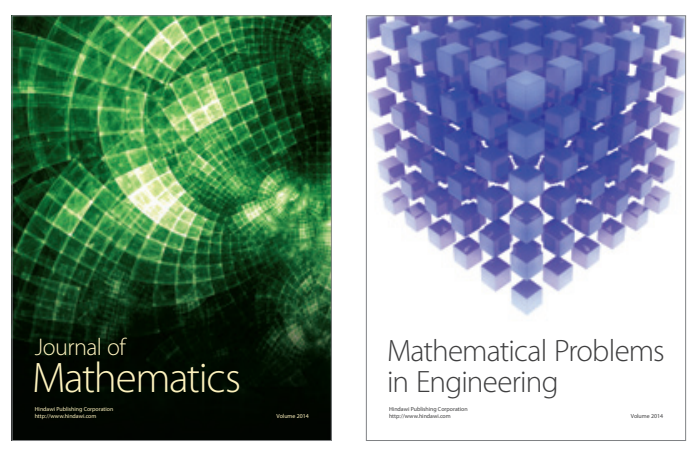

Mathematical Problems in Engineering
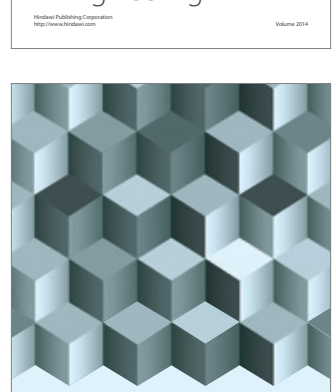

Journal of

Function Spaces
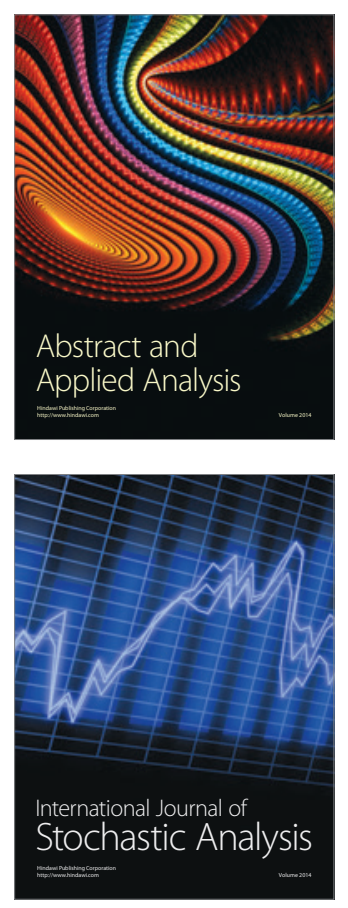

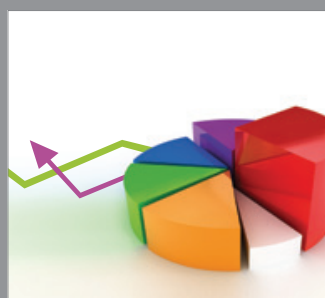

ournal of

Probability and Statistics

Promensencen
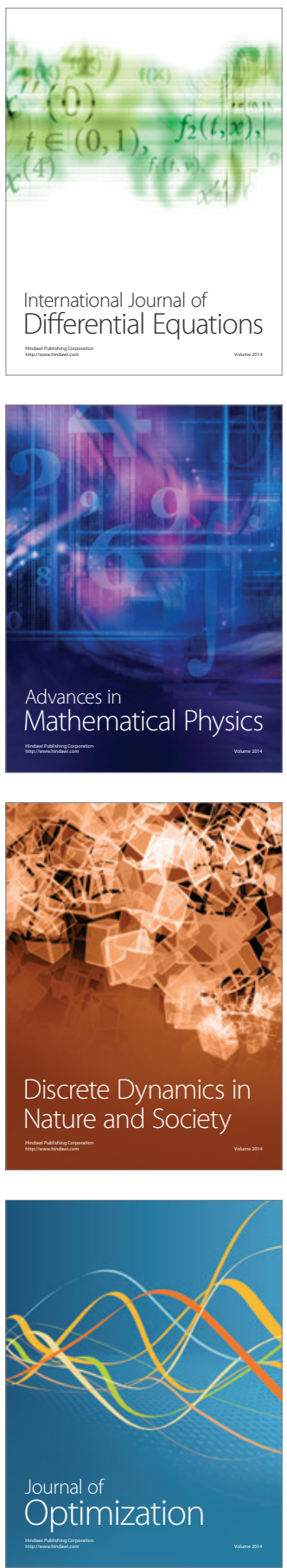